\title{
Die Doppelhalogenide von Arsen mit Cäsium und Rubidium und einige Verbindungen des Arsentrioxyds mit den Halogeniden von Cäsium, Rubidium und Kalium.
}

\author{
Von \\ H. L. WHEELER. ${ }^{1}$
}

Mit 6 Figuren im Text.

Bisher sind keine Verbindungen von Arsenhalogeniden mit Alkalihalogeniden bestimmt beschrieben. NICKLÈs ${ }^{2}$ führt in seiner Arbeit über die Bromide und Jodide des Arsens, Antimons und Wismuts an, dals diese Salze sich mit Alkali-Bromiden, bezw. -Jodiden verbinden, doch beim Arsen giebt er keine Analysen der von ihm erhalteneu Verbindungen an, und er beschreibt nicht die Methoden, die er zur Darstellung derselben benutzte. Er erwähnt sogar nicht, mit welchen Alkalihalogeniden er seine Versuche ausführte. Emmet, ${ }^{3} \mathrm{H}_{\text {arms, }}{ }^{4}$ sowie SchifF und Sestrim ${ }^{5}$ haben Verbindungen von Arsentrioxyd mit Kaliumhalogeniden beschrieben, indes ist diese Körperklasse am eingehendsten von RÜDoRFF ${ }^{6}$ studiert worden. Seine Resultate weisen auf die Existenz zweier Typen dieser Klasse von Verbindungen hin, von denen die erste 1 Mol. Alkalihalogenid auf $1 \mathrm{Mol}$. Arsentrioxyd enthält, während die andere diese Bestandteile im Verhältnis $1: 2$ besitzt. In der vorliegenden Untersuchung erhielt man die ganze Reihe der Cäsiumund Rubidium-Oxyhalogenide vom Typus $1: 1$, während die Bildung des Typus 1:2 nicht beobachtet wurde. Es zeigt sich offenbar eine Abstufung in der Beständigkeit von den Oxychloriden bis zu den Oxyjodiden, indem die Beständigkeit mit dem steigenden Atomgewicht der Halogene wächst. Versuche, Doppelhalogenide des fünfwertigen Arsens darzustellen, waren erfolglos.

1 Nach dem Manuskripte des Verfassers deutsch von Hervanv Moraht.

2 Compt. rend. 48, 839; Journ. Pharm. [3], 41, 142; Rép. Chim. pure 1, 366.

${ }^{3}$ Amer. J. science (Sill.) [1] 18, 58. - ${ }^{4}$ Ann. Pharm. Chim. 91, 371.

${ }^{5}$ Ann. Pharm. Chim. 228, 72.

- Ber. deutsch. chem. Ges. 19, 2668; 21, 3053.

z. anorg. Chem. IV. 
Die neuen Verbindungen, die in dieser Abhandlung beschrieben werden sollen, sind folgende:

$$
\begin{array}{lll}
3 \mathrm{CsCl} .2 \mathrm{AsCl}_{3} & 3 \mathrm{CsBr} .2 \mathrm{ABrr}_{3} & 3 \mathrm{CsJ} .2 \mathrm{AsJ}_{3} \\
3 \mathrm{RbCl} .2 \mathrm{AsCl}_{3} & 3 \mathrm{RbBr} .2 \mathrm{AsBr}_{3} & 3 \mathrm{RbJ} .2 \mathrm{AsJ}_{3} \\
\mathrm{CsCl} . \mathrm{As}_{2} \mathrm{O}_{3} & \mathrm{CsBr} . \mathrm{As}_{2} \mathrm{O}_{3} & \mathrm{CsJ} . \mathrm{As}_{2} \mathrm{O}_{3} \\
\mathrm{RbCl} . \mathrm{As}_{2} \mathrm{O}_{3} & \mathrm{RbBr} . \mathrm{As}_{2} \mathrm{O}_{3} & \mathrm{RbJ} . \mathrm{As}_{2} \mathrm{O}_{3} .
\end{array}
$$

Bei Versuchen, ein Doppeljodid von Kalium und Arsen darzustellen, wurde die Verbindung $\mathrm{As}_{2} \mathrm{O}_{3} . \mathrm{KJ}$ exhaltén, die von RüDorfF ${ }^{1}$ nicht beobachtet war.

Es ist hervorzuheben, dafs nur ein Typus von Doppelhalogeniden erhalten wurde. Dieser Typus entspricht dem am leichtesten darzustellenden Doppelchlorid von Cäsimm und tntimon, das Remsen und Saunders ${ }^{2}$ beschrieben haben, und dem von Cäsium und Wismut, das Remsen und Brigham ${ }^{3}$ beschrieben haben. Zahlreiche Versuche, Arsendoppelhalogenide von einem anderen Typus als diesem einzigen darzustellen, wurden angestellt, doch sind dieselben ausnahmslos erfolglos gewesen, obwohl mehrere Typen von Antimonund Wismut-Doppelhalogeniden beschrieben worden sind. An verschiedenen Beispielen haben WerLs und WHEeler ${ }^{4}$ gezeigt, dafs Cäsium- und Rubidium-Halogenide beständigere und vollständigere Doppelsalzreihen bilden, als die Halogenide der anderen Alkalimetalle. Diese Thatsache wird abermals gut bestätigt durch die Doppelhalogenide des Arsens, denn die Cäsium- und Rubidium-Doppelhalogenide lassen sich ohne Schwierigkeit darstellen, während man kein Doppelhalogenid mit Kalium erhielt.

\section{Darstellungsmethoden.}

Zur Darstellung der Doppelhalogenide ist eine stark saure Lösung nötig, um der Zersetzung des Arsenhalogenids und der daraus folgenden Bildung von Oxyverbindungen vorzubeugen. Die Doppelhalogenide sind in der starken Halogen-Säure weniger löslich, als in der verdünnten. Ein Überschufs des einen oder des anderen Bestandteils hat keinen Einflufs auf die Zusammensetzung der erhaltenen Produkte. Die Bildung von $\mathrm{As}_{2} \mathrm{O}_{3}$-Verbindungen beobachtete man beim Behandeln der Doppelhalogenide oder einer Lösung ihrer Bestandteile in starken Säuren mit Wasser oder ver-

\footnotetext{
1 Loe, eit. - ${ }^{2}$ Amer. Chem. Journ. 14, 152.

"Amer. Chem. Joum. 14, 164.

4 Amer. J. science (Sill). [3], 43, 475; 44, 42.
} 
dünnten Säuren. Die Oxyverbindungen sind schwer in verdünnten Säuren löslich, starke Säuren verwandeln die Cäsium- und RubidiumVerbindungen in die Doppelhalogenide.

\section{Analytische Methode.}

Die Salze wurden an der Pumpe filtriert und ohne Verzug sorgfältig von der Mutterlauge durch Pressen zwischen Papier befreit. Dann trocknete man sie an der Luft; in keinem Falle benutzte man Wasser zum Waschen derselben. Zur Arsenbestimmung wurde das Salz in der Kälte in Salzsäure vom spezifischen Gewicht 1.1 gelöst und etwa eine Stunde lang durch die Lösung Schwefelwasserstoff geleitet; dann fügte man etwas Alkohol hinzu und erwärmte das Ganze kurze Zeit auf dem Wasserbade, um den überschüssigen Schwefelwasserstoff zu verjagen und die Abscheidung der letzten Spuren von Arsentrisulfid zu bewirken. Das Arsensulfid wurde auf einem Gоосн-Filter gesammelt, nach dem Auswaschen mit Wasser, Alkohol und Schwefelkohlenstoff bei $100^{\circ}$ getrocknet und gewogen. Zum Filtrat fügte man Schwefelsäure und bestimmte das Alkalimetall als normales Sulfat durch Eindampfen und Glïhen des Rückstandes in einem Strom mit Ammoniak beladener Luft. Die Halogene wurden in einer besonderen Portion in der gewöhniichen Weise als Silberhalogenide bestimmt.

Die Doppelhalogenide.

Cäsium- und Rubidium-Arsenochloride: $3 \mathrm{CsCl} \cdot 2 \mathrm{AsCl}_{3}$ und $3 \mathrm{RbCl} .2 \mathrm{AsCl}_{3}$.

Dieselben besitzen eine blafsgelbe Farbe wie die entsprechenden Antimon- und Wismut-Ioppelchloride. Das Cäsiumsalz wurde durch Auflösen von $250 \mathrm{~g} \mathrm{CsCl}$ in verdünnter Salzsäure erhalten; dann fïgte man $2 \mathrm{~g} \mathrm{As}_{2} \mathrm{O}_{3}$ in verdünnter $\mathrm{HCl}$ hinzu. Dasselbe brachte einen Niederschlag hervor, der sich auf Zusatz von etwa 21 heifser Salzsäure vom spezifischen Gewicht 1,1 auflöste. Beim Erkalten schieden sich lichtgelbe Krystalle aus; ein Teil derselben wurde aus starker salzsaurer Lösung von $\mathrm{AsCl}_{3}$ umkrystallisiert. Das Rubidiumsalz wurde in derselben Weise dargestellt, aufser dafs weit stärkere Lösungen erforderlich waren. Gesättigte Lösungen von Rubidium- und Arsenchlorid in 20\% Mischen keinen Niederschlag, doch auf Zusatz von konzentrierter $\mathrm{HCl}$ scheiden sich glänzende Flitter des Doppelsalzes ab. Die Analyse dieser Produkte ergab: 


\begin{tabular}{|c|c|c|c|}
\hline \multirow{2}{*}{\multicolumn{2}{|c|}{$\begin{array}{l}\text { Mit überschüssigem } \\
\text { Cs('l dargestellt: }\end{array}$}} & \multirow{2}{*}{$\begin{array}{l}\text { Mit überschüssigem } \\
\mathrm{AsCl}_{\mathbf{3}} \text { dargestellt: }\end{array}$} & \multirow{2}{*}{$\begin{array}{l}\text { Berechnet für } \\
3 \mathrm{CsCl}_{2} 2 \mathrm{AsCl}_{3} \text { : }\end{array}$} \\
\hline & & & \\
\hline 46.14 & 45.27 & 45.09 & 45.94 \\
\hline 17.15 & $\ldots$ & 17.11 & 17.27 \\
\hline 36.89 & 36.74 & 36.12 & 36.79 \\
\hline \multicolumn{2}{|c|}{ Gefunden: } & \multicolumn{2}{|c|}{ Berechnet für $3 \mathrm{RbCl} .2 \mathrm{AsCl}_{3}$} \\
\hline $\mathrm{Rb}$ & 35.55 & \multicolumn{2}{|c|}{35.33} \\
\hline As & 20.14 & \multicolumn{2}{|c|}{20.66} \\
\hline $\mathrm{Cl}$ & 44.04 & \\
\hline
\end{tabular}

Beide Salze lassen sich aus Salzsäure vom spezifischen Gewicht 1.1 umkrystallisieren. 100 Teile $\mathrm{HCl}$ vom spezifischen Gewicht 1.2 lösen 0.429 Teile des Cäsiumsalzes und 2.935 Teile der Rubidiumverbindung auf. Da das entsprechende Kaliumsalz offenbar nicht existiert, geben diese Löslichkeiten eine geeignete Methode zur Darstellung von kaliumfreiem Cäsium und Rubidium an die Hand.

Cäsium- und Rubidium-Arsenobromide: $3 \mathrm{CsBr} .2 \mathrm{AsBr}_{3}$ und $3 \mathrm{RbBr} .2 \mathrm{AsBr}_{3}$.

Dieselben sind bernsteingelb, indem die Schattierung etwas dunkler als die der Chloride ist. Sie lassen sich am bequemsten bei Anwendung eines Überschusses des Alkalihalogenids darstellen. Man bereitete starke, heilse Lösungen der Alkalibromide in etwa $40 \%$ iger $\mathrm{HBr}$; bei Zusatz von $\mathrm{AsBr}_{3}-\mathrm{Krystallen}_{\text {schmolzen dieselben, aber }}$ erstarrten bald zu einer gelben Masse des Doppelhalogenids. Dasselbe löste sich beim Kochen, und beim Erkalten erhielt man glänzende gelbe Krystalle. - Diese Verbindungen lassen sich unveründert aus starker $\mathrm{HBr}$ umkrystallisieren; die Analyse ergab:

\begin{tabular}{|c|c|c|c|}
\hline & Gefunden: & \multirow{2}{*}{\multicolumn{2}{|c|}{ Berechnet für $3 \mathrm{CsBr} .2 \mathrm{AsBr}_{3}$ : }} \\
\hline (s & 31.91 & & \\
\hline As & 11.89 & \multicolumn{2}{|l|}{11.82} \\
\hline $\mathrm{Br}$ & 56.94 & \multicolumn{2}{|l|}{56.74} \\
\hline & lit überschüssigen & Mit überschüssigem & Berechnet für \\
\hline & $\mathrm{RbBr}$ dargestellt: & $\mathrm{AsBr}_{3}$ dargestellt: & $3 \mathrm{RbBr} .2 \mathrm{AsBr}_{3}$ \\
\hline $\mathrm{Rb}$ & 23.35 & - & 22.77 \\
\hline As & 12.55 & - & 13.31 \\
\hline $\mathrm{Br}$ & 63.97 & 64.43 & 63.92 \\
\hline
\end{tabular}

Cäsium-und Rubidium-Arsenojodide: 3 CsJ. $2 \mathrm{AsJ}_{3}$ und $3 \mathrm{RbJ} .2 \mathrm{AsJ}_{3}$.

Dieselben sind tiefrot; die gröfseren Krystalle der Cäsiumverbindung sind undurchsichtiger und erscheinen schwarz. Zur Darstellung dieser Verbindungen wurden die normalen Alkalijodide in starker, farbloser Jodwasserstoffsäure aufgelöst, worauf man diese 
Lösungen unter Kochen mit Krystallen von $\mathrm{AsJ}_{3}$ sättigte. Wenn die Jodwasserstoffsäure nicht farblos war, so war das beim Cäsiumsalz erhaltene Produkt im allgemeinen unrein, indem es mit $\mathrm{CsJ}_{3}$ gemengt war. $^{1}$

Eine gut krystallisierte Probe des Cäsiumdoppelsalzes wurde durch Darstellung des Salzes bei Gegenwart beträchtlicher Alkoholmengen erhalten. Die Analyse dieser Verbindungen ergab:

\begin{tabular}{|c|c|c|c|c|c|}
\hline Cs & 24.38 & 23.58 & $\mathrm{Rb}$ & 16.86 & 16.55 \\
\hline As & 8.92 & 8.87 & As & $\begin{array}{lll}9.96 & 10.60\end{array}$ & 9.68 \\
\hline 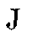 & 67.23 & 67.55 & $\mathrm{~J}$ & 73.65 & 73.77 \\
\hline
\end{tabular}

Man machte den Versuch, Kalium-Arsenochlorid durch Mischen von Lösungen von Chlorkalium und arseniger Säure darzustellen, wobei man gesättigte Lösungen dieser Körper in konzentrierter $\mathrm{HCl}$ zu diesem Zwecke anwandte. Es bildete sich dabei kein Niederschlag, und beim Einengen der Lösung schied sich Chlorkalium ab. Fügt man wässerige Chlorkaliumlösungen zu Lösungen von Arsentrioxyd in konzentrierter $\mathrm{HCl}$, so erhält man Niederschläge, die hauptsächlich ans $\mathrm{As}_{2} \mathrm{O}_{3}$ bestehen. Analoge Versuche mit Bromkalium und Arsenobromid ergaben ähnliche Resultate, und wenn man in derselben Weise mit $\mathrm{KJ}$ - und $\mathrm{AsJ}_{3}$-Lösungen in konzentrierter HJ operierte, so erhielt man nichts, als Krystalle von $\mathrm{AsJ}_{3}$ oder gemischte Ausbeuten von $\mathrm{AsJ}_{3}$ und $\mathrm{KJ}$. Ähnliche negative Resultate hinsichtlich der Bildung von Doppelhalogeniden von Ammon und Arsen hat WALLACE ${ }^{2}$ erhalten.

Verbindungen von Arsentrioxyd mit Alkalihalogeniden, $\mathrm{CsCl} . \mathrm{As}_{2} \mathrm{O}_{3}$ und $\mathrm{RbCl} . \mathrm{As}_{2} \mathrm{O}_{3}$.

Sättigte man eine heifs gesättigte wässerige Lösung von $25 \mathrm{~g}$ Chlorcäsium mit $3 \mathrm{CsCl}_{2} 2 \mathrm{AsCl}_{3}$, so bildete sich beim Erkalten ein fein verteilter weifser Niederschlag (Analyse 1). Beim Lösen von $6.5 \mathrm{~g}$ des Doppelbalogenids in $800 \mathrm{ccm}$ einer kalt gesättigten Lösung von $\mathrm{As}_{2} \mathrm{O}_{3}$ in $\mathrm{HCl}$ vom spez. Gew. 1.1 durch Frhitzen erhielt man einen ähnlichen Niederschlag (Analyse 2). Produkte von intermediärer Zusammensetzung erhielt man durch Umkrystallisieren des Doppelhalogenids aus Wasser (Analyse 3), aus 10\% iger $\mathrm{HCl}$ (Analyse 4) und aus 15\% iger $\mathrm{HCl}$ (Analyse 5).

\footnotetext{
1 Amer J. science (Sill.) [3], 43, 17.

${ }^{2}$ Phil. Mag. [4], 16, 358; [4], 17, 122, 261.
} 


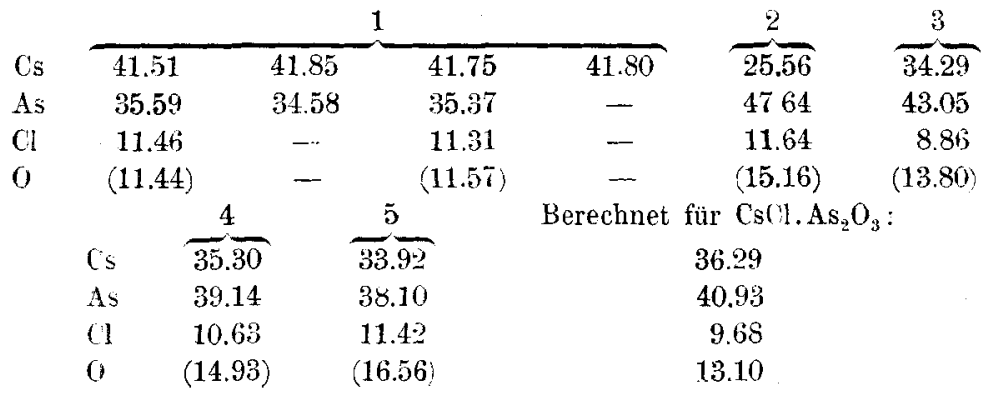

Die Analysen zeigen eine beträchtliche Abweichung von der nach der Formel verlangten Zusammensetzung, indem die unter den verschiedensten Bedingungen dargestellten Produkte als Verhältnis von Arsentrioxyd zu Chlorcäsium $3: 4$ und $3: 2$ anstatt $1: 1$ lieferten. Indes wechselten die Bedingungen so sehr, dals es gerechtfertigt erscheint, die Existenz einer Verbindung vom Typus $1: 1$ anzunehmen.

Krystallisierte man das Rubidium-Doppelhalogenid aus etwa $15 \%$ iger $\mathrm{HCl}$ um, so erhielt man bei langsamem Erkalten eine weifse krystallinische Kruste. Dieselbe lieferte anlaytische Resultate, welche mit der Formel RbCl. $\Lambda s_{z} O_{i}$ ïbereinstimmen.

$\begin{array}{lcc} & \text { Gefunden: } & \text { Berechnet für } \mathrm{RbCl} \cdot \mathrm{As}_{2} \mathrm{O}_{3} \text { : } \\ \mathrm{Rh} & 26.90 & 26.80 \\ \mathrm{As} & - & 47.03 \\ \mathrm{Cl} & 11.41 & 11.13 \\ \mathrm{O} & - & 15.04\end{array}$

Unter dem Mikroskop erscheinen diese Verbindungen als umregelmärsige Körnchen oder Plättchen von unbestimmter Krystallform.

$\mathrm{CsBr} \cdot \mathrm{As}_{2} \mathrm{O}_{3}$ und $\mathrm{RbBr} \cdot \mathrm{As}_{2} \mathrm{O}_{3}$. - Krystallisiert man die Doppelbromide aus Wasser oder verdünter $\mathrm{HBr}$ um, so liefern sie diese Oxyrerbindungen, und diese scheiden sich im allgemeinen in Gestalt einer weilsen Kruste am Boden und an den Wänden des Gefälses ab. Die Analyse solcher Produkte ergab:

$3 \mathrm{CsBr}_{2} 2 \mathrm{AsBr}_{3}, \quad 3 \mathrm{CsBr}_{2} 2 \mathrm{AsBr}_{3}$, aus $\quad$ Berechnet für

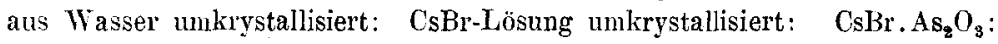

$\begin{array}{lccc}\text { Cs } & 32.42 & - & 32.36 \\ \mathrm{As} & 36.52 & - & 36.50 \\ \mathrm{Br} & 19.57 & 19.59 & 19.46 \\ \mathrm{O} & (11.49) & - & 11.68 \\ & 3 \mathrm{RbBr} .2 \mathrm{AsBr}_{3}, \text { aus } & 3 \mathrm{RbBr} .2 \mathrm{AsBr}_{3}, \text { aus } & \text { Berechnet für } \\ & \text { verdiunnter } \mathrm{HBr} \text { umkrystallisiert: } & \text { Wasser umkrystallisiert: } & \mathrm{RbBr} . \mathrm{As}_{2} \mathrm{O}_{3} \text { : } \\ \mathrm{Rb} & 24.24 & 16.56 & 23.52 \\ \mathrm{As} & 40.06 & 50.74 & 41.27 \\ \mathrm{Br} & 24.53 & 15.91 & 22.01 \\ \mathrm{O} & (11.17) & (16.79) & 13.20\end{array}$


Es mufs hervorgehoben werden, dafs das durch Umkrystallisieren von $3 \mathrm{RbBr} .2 \mathrm{AsBr}_{3}$ aus Wasser erhaltene Produkt unrein ist, während die auf dieselbe Weise dargestellte Cäsiumverbindung der Formel entspricht. Es ist dies eine Illustration für die grölsere Neigung der Cäsiumbalogenide zur Bildung von Doppelsalzen, als der Rubidiumhalogenide. Diese beiden Verbindungen sind weifs, doch wird die Rubidiumverbindung beim Trocknen etwas gelb. Unter dem Mikroskop sah man bei der Cäsiumverbindung, bei der die Lösung langsam erkaltet war, sechsseitige Plättchen; ferner beobachtete man hexagonale Krystalle mit kurzsäuligem rhomboedrischen Habitus; sie waren einaxig mit schwacher negativer Doppelbrechung. Die Rubidiumverbindung wurde ebenfalls in hexagonalen Krystallen erhalten, die rhomboedrische Symmetrie und schwache negative Doppelbrechung zeigten.

CsJ.As, $O_{3}$, RbJ.As, $O_{3}$ und KJ. $A_{2} O_{3}$. - Die Bildung dieser Verbindungen wurde beobachtet, wenn verdünnte jodwasserstoffsaure Lösungen der Alkalijodide mit verdünnten sauren Lösungen von $\mathrm{AsJ}_{3}$ vermischt wurden. Mischte man die Lösungen in der Hitze, so schieden sich diese Doppelsalze beim Erkalten in Form krystallinischer gelber Krusten am Boden und an den Wänden des Gefäfses ab. Diese Krystalle sind im allgemeinen etwas gröfser, als die der Verbindungen von $\mathrm{As}_{2} \mathrm{O}_{3}$ mit den Chloriden und Bromiden. Unter dem Mikroskop zeigten sie die Form sechsseitiger Plättchen; dieselben zeigen eine starke negative Doppelbrechung. Die Kaliumverbindung erschien ebenfalls in Form sechsseitiger Plüttchen; dieselben blieben beim Drehen zwischen gekreuzten Nikols dunkel. Sie waren zu klein, um eine Axenfigur zu liefern; die gröfsten Plättchen überschritten nicht einen Durchmesser von $0.01 \mathrm{~mm}$; wahrscheinlich sind sie hexagonal. Die Analyse ergab:

$\begin{array}{lcc} & \text { Gefunden: } & \text { Berechnet für CsJ. } \mathrm{As}_{2} \mathrm{O}_{3} \text { : } \\ \mathrm{Cs} & 29.31 & 29.04 \\ \mathrm{As} & 32.01 & 32.75 \\ \mathrm{~J} & 28.94 & 27.73 \\ \mathrm{O} & (9.74) & 10.48 \\ & & \text { Berechnet für } \mathrm{RbJ} . \mathrm{As}_{2} \mathrm{O}_{3} \text { : } \\ \mathrm{Rb} & 20.35 & 20.83 \\ \mathrm{As} & 36.78 & 36.54 \\ \mathrm{~J} & 31.94 & 30.93 \\ \text { O } & (10.93) & \mathbf{1 1 . 7 0} \\ & & \mathbf{1 0 . 7 4} \\ \mathrm{K} & 10.75 & 41.20 \\ \mathrm{As} & 42.85 & 34.88 \\ \mathrm{~J} & 34.13 & \mathbf{1 3 . 1 8} \\ \mathrm{O} & (12.27) & \text { Berechnet }\end{array}$


Krystallographie.

Die Krystallform der Cäsium- und Rubidium-Arsenohalogenide ist hexagonal; alle wurden gemessen und als isomorph gefunden. Im allgemeinen war der Habitus holoedrisch, obwohl er beim Cäsiumarsenobromid rhomboedrisch war. Alle diese Salze zeigen eine ausgesprochene Spaltbarkeit nach der Basis, und zu dieser parallele Plättchen erwiesen sich bei Untersuchung mit dem Stauroskop als einaxig. Die Doppelchloride und -bromide zeigen eine schwache negative Doppelbrechung, während die Doppeljodide positiv sind. Die beobachteten Formen sind folgende:

\begin{tabular}{rrr|rrr}
$c$ & 0001 & $0 \mathrm{P}$ & $r$ & $10 \overline{1} 1$ & $\mathrm{R}$ \\
$a$ & $11 \overline{2} 0$ & $\infty \mathrm{P}_{2}$ & $r$ & $01 \overline{1} 1$ & $-\mathrm{R}$ \\
$m$ & $10 \overline{1} 0$ & $\infty \mathrm{R}$ & $p$ & $20 \overline{1} 1$ & $2 \mathrm{R}$
\end{tabular}

Die steile Pyramide $p$ wurde allein bei den Jodiden gefunden. Die folgende Tabelle giebt die Längen der Vertikalaxen an:

\begin{tabular}{|c|c|c|}
\hline & a & $\mathrm{c}$ \\
\hline $3 \mathrm{CsCl} .2 \mathrm{AsCl}_{3}$ & 1 & 1.209 \\
\hline $3 \mathrm{RbCl} .2 \mathrm{AsCl}_{3}$ & 1 & 1.210 \\
\hline $3 \mathrm{CsBr} \cdot 2 \mathrm{AsBr}_{3}$ & 1 & 1.219 \\
\hline $3 \mathrm{RbBr} .2 \mathrm{AsBr}$ & 1 & 1.220 \\
\hline $3 \mathrm{CsJ} .2 \mathrm{AsJ}_{3}$ & 1 & 1.244 \\
\hline $3 \mathrm{RbJ} .2 \mathrm{AsJ}_{3}$ & 1 & 1.243 \\
\hline
\end{tabular}

Eine Vergleichung der obigen Axenverhältnisse zeigt die interessante Thatsache, dafs der Ersatz von Cäsium durch Rubidium keinen merklichen Einflufs auf die Axenlängen ausübt, und dafs in dieser Reihe die Vertikalaxen wachsen, wie das Atomgewicht des Halogens steigt. Die Krystalle waren genügend beständig, um gute Messungen zu erhalten, obwohl sie gewöhnlich bei langem Luftaussatz ihren Glanz verlieren. In den Messungstabellen sind die als fundamental ausgewählten Winkel durch einen Stern bezeichnet.

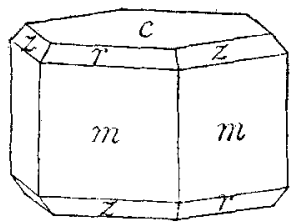

Fig. 1

$3 \mathrm{CsCl} .2 \mathrm{AsCl}_{3}$. Dieses Salz wurde in Krystallen von etwa $1-2 \mathrm{~mm}$ Durchmesser dargestellt; die beobachteten Formen sind $m, a$, $r, z$ und $c$. Man suchte sorgfältig nach Anzeichen für eine rhomboedrische Entwickelung der Flächen $r$ und $z$, fand aber keine. Offenbar sind sie stets holoedrisch in ihrer Aus-

bildung (Fig. 1). 


\begin{tabular}{|c|c|c|c|c|}
\hline$m$ & $\hat{c} c$ & $1010 \wedge 0001$ & $\begin{array}{l}\text { Gemessen: } \\
90^{\circ}\end{array}$ & $\begin{array}{c}\text { Berechnet } \\
90^{\circ}\end{array}$ \\
\hline 0 & $\sim r$ & 0001 ^ $10 \overline{11}$ & $\$ 54^{0} \quad 24^{\prime}$ & -- \\
\hline$y$ & ᄉ $m$ & $10 \overline{1} 1$ 人 $10 \overline{10}$ & $35^{0} 39^{\prime}$ & $35^{0} 36^{4}$ \\
\hline$m$ & $\wedge z$ & $1010 \curvearrowright 0111$ & $66^{\circ} 3^{\prime}$ & $66^{0} 1^{\circ}$ \\
\hline & ᄉ $z$ & $10 \overline{11} \curvearrowright 0111$ & 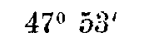 & $47^{\circ} 58^{\prime}$ \\
\hline
\end{tabular}

Eine ziemlich geringe Spaltbarkeit nach dem Prisma ist vorhanden, und dazu parallele Plättchen zeigen parallele Auslöschung.

$3 \mathrm{RbCl} .2 \mathrm{AsCl}_{3}$. Dieses Salz wurde in Krystallen bis zu einem Durchmesser von etwa $5 \mathrm{~mm}$ hinauf dargestellt; die beobachteten Formen sind $c, m, r$ und $z$. Die Flächen $r$ und $z$ waren selten vorhanden, doch, wenn sie auftraten, konnte man nicht sehen, ob sie rhomboedrische Symmetrie zeigten oder nicht. Einander durchwachsende Zwillinge sind häufig, wobei die Zwillingsebene das Rhomboeder $0 \overline{1} 11$ ist (Fig. 2).

$$
\begin{array}{llllc}
c \sim c \text { (Zwilling) } 0001 & \sim 0001 & * 71^{\circ} 3^{\prime} & - \\
c \wedge c & 0001 & \sim 10 \overline{1} & 54^{\circ} 21^{\prime} & 54^{\circ} 28^{1} \mathrm{~s}^{\prime} \\
m \sim r & 10 \overline{10} & \sim 10 \overline{1} 1 & 35^{\circ} 39^{\prime} & 35^{\circ} 31^{1} \mathbf{2}^{\prime}
\end{array}
$$

$\begin{array}{cc}\text { Gemessen: } & \text { Berechnet: } \\ 71^{\circ} 3^{\prime} & - \\ 54^{0} 21^{\prime} & 54^{0} 28^{1} /^{\prime} \\ 35^{0} 39^{\prime} & 35^{\circ} 31^{1 / 2^{\prime}}\end{array}$

Bei der Untersuchung dieses Salzes in konvergentem polarisierten Licht sieht man ein einaxiges Kreuz, dessen Arme nicht schwarz sind, sondern ein tiefes und glänzendes Blau besitzen, während der Charakter negativ ist. Bei Untersuchung in monochromatischem roten Licht sind die Krystalle nahezu isotrop, wobei die Doppelbrechung äufserst schwach und wahrscheinlich negativ ist. In blauem Licht indes sieht man ein deutliches Kreuz, begleitet von axialen Ringen. Dieser Unterschied zwischen Rot und Blau erklärt das gefärbte Kreuz, das man im weifsen Licht sieht. Zur Axe $c$ parallele Schnitte zeigen das tiefe eigentümliche Blau, das für einaxige Körper mit den oben erwähnten optischen Eigenschaften charakteristisch ist.

$3 \mathrm{CsBr} .2 \mathrm{AsBr}_{3}$. Dieses Salz wurde in Krystallen bis zu $1^{1 / 2} \mathrm{~mm}$ Durchmesser hinauf dargestellt; die beobachteten Formen sind $c, m, r$ und $z$. Es ist das einzige Salz der Reihe, das einen rhomboedrischen Habitus besitzt, und da der Winkel des Rhomboeders nahezu $90^{\circ}$ ist, so sehen die Krystalle wie Würfel aus. Figur 3 zeigt eine ideale Kombination von $r$ mit $m, z$ nnd $c$; diese Form wurde nicht beobachtet, da

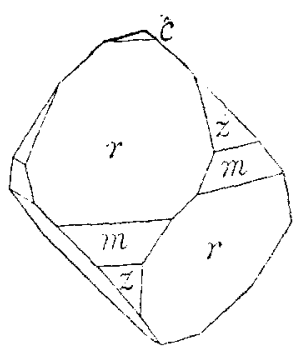

Fig. :. 


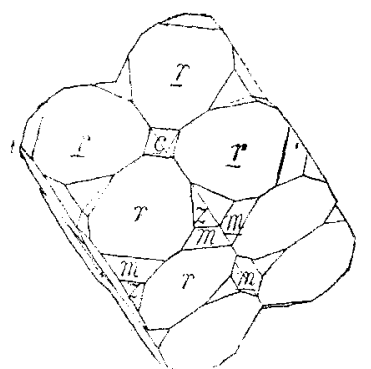

Fig. 4.

die Krystalle ausnahmslos Zwillinge sind. Eine ideale Darstellung des Zwillings ist in Figur 4 gegeben. Die Flächen $r$ waren so gekrümmt und gefurcht, dafs daran keine genauen Messungen angestellt werden komuten.

$$
\begin{array}{llcc} 
& \text { Gemessen: } & \text { Berechnet: } \\
m \wedge z & 0110 \frown 0111 & * 35^{0} 23^{\prime} & - \\
z \wedge z & 10 \overline{11} \frown \overline{1} 101 & - & 89^{\prime} 50^{\prime}
\end{array}
$$

$3 \mathrm{RbBr} .2 \mathrm{AsBr}_{3}$. - Dieses Salz wurde in kleinen Krystallen bis zu $2 \mathrm{~mm}$ Durchmesser dargestellt; die beobachteten Formen waren $c, m$ und $r$. Die Krystalle wurden in zweierlei Gestalt dargestellt: bei der Darstellung mit einem Überschufs von RbBr scheidet sich das Salz in prismatischen Krystallen aus, welche in der Form dem Caeciumarsenochlorid gleichen (Fig 1). Bei einem Versuch bei

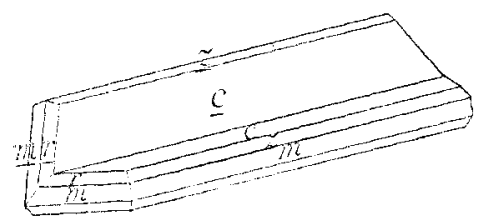

Fig. 5.

$\begin{array}{ll}c \sim c \text { (Zwilling) } & 0001 \sim 0001 \\ c \wedge t & 0001 \sim 1011 \\ r \wedge m & 1011 \sim 1010 \\ r \sim z & 1011 \sim 0111 \\ m \wedge z & 1010 \sim 0111\end{array}$
Gegenwart yon $\mathrm{AsBr}_{3}$ erhielt man Zwillinge; hier ist die Zwillingsebene die gleiche Rhomboederfläche, wie in Figur 2; jedoch sind einige Flächen parallel der Kante zwischen den beiden Basenebenen verlängert (Fig. 5).

3CsJ . $2 \mathrm{AsJ}_{3}$. - Dieses Salz wurde in schönen Krystallen bis zu 3 mm Länge dargestellt; die beobachteten Formen sind $p$ und $c$. Der Habitus ist der einer steilen, doppelt begrenzten hexagonalen Pyramide mit kleinen oder ganz fehlenden Basenebenen. Oft sind die mittleren Kanten abgerundet durch wellenartige Kombinationen der Pyramiden, wodurch Anlals zu horizontaler Furchung gegeben ist. Kein Anzeichen für ein Prisma oder eine rhomboedrische Ausbildung der Pyramidenflächen war vorhanden (Fig. 6).

Fig. 6.

$\begin{array}{cc}\text { Gemessen: } & \text { Berechnet: } \\ 70^{\circ} 45^{\prime} & 70^{\circ} 44^{\prime} \\ 54^{0} 40^{\circ} & 54^{0} 38^{\prime} \\ * 35^{\circ} 22^{\prime} & - \\ 48^{\circ} 14^{\prime} & 48^{\circ} 77^{\prime} / 2^{\prime} \\ 65^{\circ} 34^{\prime} & 65^{0} 56^{\prime}\end{array}$

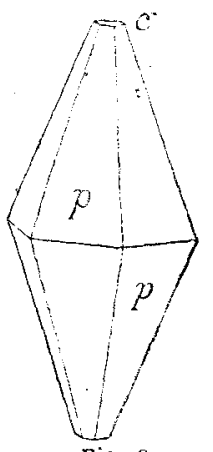

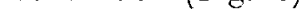

$\begin{array}{ll}c \wedge p & 0001 \sim 20 \overline{1} 1 \\ p \wedge p & 2021 \sim 20 \overline{21} \\ p \wedge p & 20 \overline{2} 1 \sim 02 \overline{2} 1\end{array}$

Gemessen: Berechnet:

$=70^{\circ} 49 \quad-$

$38^{\circ} 19^{\circ} \quad 38^{\circ} 22^{\prime}$

$56^{\circ} 24^{1 / 2^{\prime}} \quad 56^{0} 21^{\prime} 34^{\prime \prime}$ 
Dieses Salz besitzt keine optischen Anomalien; Pleochroismus liefs sich nicht beobachten.

$3 \mathrm{RbJ} .2 \mathrm{AsJ}_{3}$. - Dieses Salz wurde in sehr kleinen Krystallen von nicht über $1 \mathrm{~mm}$ Länge dargestellt; die beobachteten Formen waren $c, m$ und $p$. Der Habitus ist ähnlich Figur 6 , doch sind die Mittelkanten gewöhnlich durch die Flächen des Prismas $m$ ersetzt oder durch horizontale Furchung abgerundet.

$\begin{array}{llll} & & \text { Gemessen: } & \text { Berechnet: } \\ p \wedge p & 2021 \text { ^ } 0221 & * 56^{\circ} 21^{\prime} & - \\ c \wedge p^{\prime} & 0001 \frown 2021 & 70^{\circ} 47^{\prime} & 70^{\circ} \\ p \frown m & 2021 \frown 1010 & 19^{\circ} 12^{\prime} & 20^{\circ} 56^{\prime}\end{array}$

Optisch zeigt dieses Salz Anomalien. Basale Spaltplïttchen sind zwischen gekreuzten Nicols nicht dunkel, sondern hell, und bleiben es beim Drehen. Im konvergenten Lichte sieht man den Ort einer optischen Axe im Centrum des Feldes, zusammenfallend mit der Vertikalaxe $\dot{c}$. Die dieser Axe zunächst liegende Bisektrix ist die der geringsten Elasticität c. Das Salz ist indes nicht eigentlich zweiaxig, da die Ebene der optischen Axen bisweilen parallel, bisweilen senkrecht zur Prismenkante steht. Aufserdem wechselt diese Richtung häufig in demselben Plättchen von Stelle zu Stelle, und bisweilen sieht man keinen Balken, sondern einen schwarzen Fleck, ungeben ron Ringen, im Centrum des Feldes. Ein solches Verhalten lärst sich durch die Annahme erklären, dafs sich die Krystalle in einem Zustande inmerer Spannung befinden. Dem Prisma parallele Schnitte bleiben bisweilen zwischen gekreuzten Nikols hell, bisweilen werden sie bei wechselnden Winkeln dunkel und zeigen einen geringen Pleochroismus, wobei die Absorption $\varepsilon>\omega$ und die Farbe ein tiefes Orangerot ist.

Zum Schlusse möchte der Verfasser Herrn Prof. H. L. WeLrs für wertvolle Ratschlage, die vorliegende Untersuchung betreffend, seinen Dank aussprechen, und ebenso Herrn Prof. S. L. PEnField, unter dessen Leitung die Krystallographie dieser Salze untersucht wurde. Ferner ist der Verfasser Herrn L. V. Prrsson für seine Unterstiitzung bei der optischen Beschreibung dieser Salze verpflichtet.

Sheffeld Scientific School, New Hacen, Conn., März 1893.

Bei der Redaktion eingegangen am 23. Mai 1893. 